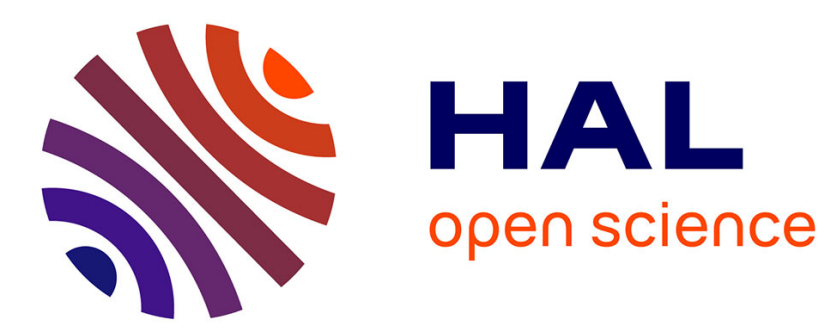

\title{
La loi 1901 faute de mieux. Les Républicains face a l'association au tournant du XXe siècle
}

\author{
Chloé Gaboriaux
}

\section{To cite this version:}

Chloé Gaboriaux. La loi 1901 faute de mieux. Les Républicains face a l'association au tournant du XXe siècle. The Tocqueville Review/La revue Tocqueville, 2011, 32 (2), pp.53-65. 10.1353/toc.2011.0014 . halshs-03421337

\section{HAL Id: halshs-03421337 https://shs.hal.science/halshs-03421337}

Submitted on 17 Dec 2021

HAL is a multi-disciplinary open access archive for the deposit and dissemination of scientific research documents, whether they are published or not. The documents may come from teaching and research institutions in France or abroad, or from public or private research centers.
L'archive ouverte pluridisciplinaire HAL, est destinée au dépôt et à la diffusion de documents scientifiques de niveau recherche, publiés ou non, émanant des établissements d'enseignement et de recherche français ou étrangers, des laboratoires publics ou privés. 
The Tocqueville Review/La Revue Tocqueville, Vol. XXXII, $n^{\circ} 2$ - 2011

\section{LA LOI 1901 FAUTE DE MIEUX LES RÉPUBLICAINS FACE A L'ASSOCIATION AU TOURNANT DU XX' SIÈCLE}

Chloé GABOriauX

L'un des nombreux paradoxes de la France du XIXe siècle est sans doute d'avoir encensé et pratiqué la liberté d'association sans pourtant parvenir à l'inscrire dans la loi, restée très répressive en la matière. Il a ainsi fallu attendre 1901 pour qu'enfin elle entre dans le droit français. Elle est aujourd'hui généralement saluée comme une victoire de la liberté, enfin arrachée à la réaction par des républicains qui la réclamaient depuis si longtemps. De nombreux auteurs en ont cependant montré les limites: outre le statut d'exception donné aux congrégations religieuses ${ }^{1}$, ils ont souligné les restrictions apportées à la capacité juridique des associations, accordée de façon graduée en fonction de leur reconnaissance par l'Etat - inexistante pour les associations non déclarées, réduite pour les associations déclarées aux cotisations, local et immeubles strictement nécessaires à l'accomplissement de leur but, élargie pour les associations reconnues d'utilité publique. Elles renvoient pour Jean-Pierre Machelon à une « conception fort étriquée» de la liberté d'association, signe que «le temps du refus » n'est pas tout à fait terminé en 19012. Parce qu'elle lésine sur les moyens offerts aux associations pour se développer, Pierre Rosanvallon peut dire de la loi 1901 qu'elle « ne connait que l'acte d'association et [qu']elle ignore l'institution qui résulte de cette opération $»^{3}$. Dans cette perspective, la méfiance bien française à l'égard des corps intermédiaires paraît encore forte chez les artisans de la loi 1901. 
C'est ce constat qu'on voudrait réinterroger ici à partir des réflexions que les républicains tiennent autour de 1901 non pas au Parlement mais au-dehors, dans les revues politiques et au sein des associations dont ils font partie. L'une des particularités du moment 1901, bien mise en valeur par le colloque Associations et champ politique organisé au Sénat en novembre 2000, réside en effet dans l'implication des élites politiques républicaines dans la vie associative foisonnante que connaît la France depuis les années 18804. Or les réflexions qu'ils y développent mettent en évidence l'importance de l'association comme institution justement, jouant un rôle essentiel entre l'individu d'une part et l'Etat d'autre part. Elles conduisent donc à remettre en question l'idée selon laquelle l'individualisme républicain impliquerait une reconnaissance a minima des associations (I). Mais il faut alors s'interroger sur l'absence de concrétisation juridique de ces conceptions. A un moment où la vigueur retrouvée de l'anticléricalisme permet de régler, sous un titre spécial, le problème des congrégations, pourquoi la loi 1901 maintient-elle les restrictions évoquées plus haut? La lutte contre les associations religieuses a-t-elle conduit les républicains à renouer avec leurs vieilles réticences à l'égard des corps intermédiaires ? (II) L'hypothèse qu'on proposera pour finir est qu'elle les a plutôt amenés à renoncer provisoirement à leurs ambitions en matière d'association, les dispensant par là même de les confronter aux exigences du pluralisme démocratique (III).

\section{L'ASSOCIATION AU CEUR DU PROJET REPUBLICAIN}

Le ralliement des républicains à la liberté d'association au cours du XIX $^{\mathrm{e}}$ siècle est bien souvent présenté comme un "compromis» ${ }^{5}$. Traditionnellement attachés à une définition de l'intérêt général et de la liberté individuelle qui les a longtemps conduits à bannir tout corps intermédiaire entre l'individu et les pouvoirs publics, les républicains auraient tenté d'adapter leurs conceptions aux enjeux intellectuels et sociopolitiques du moment: la naissance de la sociologie les invitait d'une part à admettre le rôle social des groupements ; la multiplication de fait des associations ne cessait d'autre part de creuser l'écart entre les pratiques sociales et le droit. La loi de 1901 relative au contrat d'association témoignerait de l'« essence » de ce compromis ${ }^{6}:$ qu'on s'en félicite comme Jean-Louis Bardout ${ }^{7}$ ou qu'on le regrette comme Pierre Rosanvallon', la liberté d'association y apparait comme purement individuelle, contractuelle et surtout garantie et encadrée par l'Etat. 
L'analyse des réflexions que les républicains tiennent au sein des innombrables associations dont ils font partie et qu'ils rendent régulièrement publiques dans la presse et les revues politiques tend pourtant à remettre en question le terme de «compromis » comme la démarche qui a permis de le définir. Elle montre en effet que la défense de la liberté d'association relève d'une véritable redéfinition du rapport privé/public, qui tient moins du compromis que d'un remaniement profond des conceptions républicaines, et qui ne saurait donc trouver son illustration dans la loi de 1901.

Au cours du XIXe siècle, la conception républicaine de la citoyenneté a en effet connu un infléchissement majeur. D'abord pensée comme prenant naissance dans le face à face de l'individu avec l'Etat, elle a progressivement intégré un troisième terme: le regroupement volontaire, qui seul paraissait à même de susciter le citoyen dans l'individu, en le conduisant à se décentrer et à prendre ainsi peu à peu conscience de la collectivité. Les termes psychosociologiques dans lesquels ce processus est décrit par les républicains depuis le Second Empire sont certes tributaires des sciences sociales alors en plein essor. Mais leur nouvel intérêt pour l'association n'est pas purement théorique. Elle se nourrit du spectre du bonapartisme qui, aux yeux des républicains, n'a pu être instauré et durer qu'en vertu de l'isolement des individus, où ils voient, après Tocqueville, le terrain privilégié du despotisme: tant qu'ils vivent repliés sur eux-mêmes, séparés les uns des autres, les individus ne peuvent que difficilement se former une idée de l'intérêt général et, même lorsque c'est le cas, ils ne sauraient trouver la force nécessaire pour résister à un éventuel usurpateur' ${ }^{9}$. La question sociale vient confirmer l'importance de l'association ainsi conçue : elle permet de faire vivre la fraternité sans rompre avec le primat de l'intérêt général et protège ainsi les plus faibles tout en les intégrant à la nation. C'est ce que résume parfaitement le président du Cercle caennais de la Ligue de l'Enseignement, invité quelques mois avant le vote de la loi 1901 à s'exprimer sur le sujet dans une conférence organisé par l'Association républicaine de Caen : "prohiber plus longtemps l'association, ce serait préparer la dictature ou l'anarchie - la dictature d'autant plus redoutable qu'elle pourrait toujours s'établir sans avoir à lutter contre la résistance de forces disciplinées ; l'anarchie lorsque les individus se sentant libres de toute compression et affranchis de toute autorité vraiment forte ne sauraient que s'agiter chacun pour soi dans 
une désastreuse confusion ${ }^{10} »$.

Dans cette perspective, l'association doit se tenir à l'interface du privé et du public, à égale distance de l'individu et de l'Etat. Elle ne saurait donc se réduire à la somme des individus qui la composent. L'enjeu en effet pour les animateurs des associations républicaines, c'est d'abord d'opérer le passage du privé au public, des intérêts particuliers à l'intérêt général. "Une association, écrit en 1896 Léon Bourgeois, alors président de la Ligue de l'Enseignement, c'est l'apprentissage de la vie sociale, parce que c'est l'habitude donnée à un certain nombre d'hommes de penser à autre chose qu'à euxmêmes. Quelle que soit l'association, que ce soit une société d'instruction ou une société d'éducation civique ou politique, son but principal, c'est d'apprendre chaque jour davantage à se sacrifier au but commun et c'est faire sur un petit terrain, sur un petit espace, dans un petit domaine, l'image réduite, visible pour quelques yeux, de ce que doit être la grande société humaine dans laquelle le but essentiel sera de penser aux autres au lieu de penser à soi $^{11}$.» Or pour former «l'image réduite» de la communauté nationale, il faut que l'association puisse constituer une entité collective dotée des moyens de son développement. Sur ce point, tous les républicains sont d'accord, même s'ils butent sur la question d'accorder ou non, et dans quelle mesure, la personnalité civile aux associations. L'argumentation développée par Yves Guyot en 1898 dans la Revue politique et parlementaire est à ce titre éclairant: s'il refuse quant à lui de leur donner la personnalité civile, il condamne en revanche toute « restriction au droit de posséder $»^{12}$.

La question des moyens offerts à l'association est d'autant plus importante aux yeux des républicains qu'elle doit dans leur esprit pouvoir résister à un éventuel usurpateur. Il s'agit en effet non seulement de faire naitre et consolider l'intérêt général dans le cœur des citoyens mais aussi de leur donner la force de sauver les institutions républicaines le jour où elles seraient menacées. Cette dernière fonction attribuée aux associations implique donc aussi de préserver leur indépendance à l'égard de l'Etat, au cas où lui-même serait dévié de sa mission. La position des animateurs de la Ligue de l'Enseignement est encore une fois un bon exemple des conceptions républicaines de l'association ${ }^{13}$. Comme Jean Macé, Léon Bourgeois, qui lui succède, rappelle que l'Etat doit rester à distance : «C'est un peuple libre dont l'éducation est en cause. C'est dans la liberté qu'il 
faut le préparer à vivre dans la liberté14 $»$. A cet égard, il faut se garder de confondre intérêt général et Etat. Ce n'est pas parce que l'association devient dans l'esprit des républicains le «relais de la généralité $»^{15}$ qu'elle admet la tutelle de l'Etat: au contraire, elle doit s'en prémunir pour préserver sa fonction critique, qui s'exercera au besoin contre l'Etat.

Véritable entité collective dotée du droit de posséder et jalouse de son indépendance à l'égard de l'Etat, le portrait de l'association idéale tel qu'il ressort des débats et discours tenus par les républicains est donc loin du rôle finalement accordé aux associations dans la loi de 1901. Et pour cause : comme l’a rappelé Gilles Le Béguec, la réflexion riche et dense menée depuis plus de dix ans dans le monde intellectuel et politique «n'a pas été sérieusement prise en compte, de manière explicite tout au moins, au cours de la discussion [parlementaire] du printemps $1901 »$, toute entière absorbée par la question religieuse ${ }^{16}$.

\section{LE PÉRIL CLÉRICAL}

On le sait, la question religieuse a longtemps freiné l'inscription dans la loi de la liberté républicaine. Comme le dit très bien Charles Floquet en mai 1881, les républicains pourtant attachés à la liberté d'association ont été arrêtés dans leurs projets de loi «par l'embarras d'organiser et de garantir la liberté d'association sans autoriser la licence des congrégations ${ }^{17} »$. Comment en effet accorder aux associations les moyens de leur développement et de leur indépendance à l'égard de l'Etat sans donner en même temps une arme puissante à leurs adversaires ?

La solution d'un régime séparé pour les congrégations avait d'abord paru peu conforme aux valeurs républicaines. Dans son rapport sur la proposition de loi Dufaure, déposé le 27 juin 1882, Jules Simon l'avait dit avec force: "nous fondons une liberté nouvelle, allons-nous établir entre ceux qui en jouiront des catégories? " La question était selon lui tranchée par le principe d'égalité devant la loi, qui exigeait un "droit commun", une "règle commune », mais aussi par le « respect pour la liberté de conscience, qui ne permet[tait] pas de faire acception de l'opinion des citoyens » pour organiser un statut d'exception ${ }^{18}$. 
Mais au tournant du $\mathrm{XX}^{\mathrm{e}}$ siècle, le regain d'anticléricalisme qui anime la gauche républicaine après l'Affaire Dreyfus balaie les derniers scrupules. Les parlementaires s'acheminent alors vers un traitement spécial des congrégations, que ses promoteurs justifient par une argumentation fondée sur la différence de nature qui séparerait l'association de la congrégation, souvent identifiée à la corporation. Les discours de son principal artisan, Pierre WaldeckRousseau, d'ailleurs réunis dès 1901 sous le titre Associations et congrégations en témoignent ${ }^{19}$. Ils opposent en effet les deux types de groupement terme à terme: la congrégation repose sur des vœux quand l'association respecte la liberté de ses membres; le groupe prime sur l'individu dans la première tandis que la seconde donne la priorité à l'individu sur le groupe; la congrégation impose la communauté de vie alors que l'association offre des moments partagés non exclusifs d'autres sociabilités, privilégiant un engagement partiel sur l'engagement total exigé par la congrégation. Cette description antithétique fonde aux yeux des avocats du projet de loi les caractéristiques légales de l'association : durée limitée dans le temps par opposition à la durée illimitée des congrégations, propriété collective réduite aux stricts besoins de l'association déclarée par opposition à la « mainmorte » tant dénoncée dans la congrégation.

C’est ce qui fait dire à Pierre Rosanvallon que la société civile en France «n'a pas été pensée dans sa différence à l'Etat mais dans sa rupture avec le monde religieux », renouant ainsi avec une "vision archaïque » très proche de celle du XVII e siècle ${ }^{20}$. La loi de 1901 relative au contrat d'association illustrerait alors parfaitement l'incapacité des républicains français à penser l'autonomie de la société civile, toujours rabattue sur des libertés purement individuelles conquises et garanties par l'Etat contre l'Eglise.

L'affirmation semble pourtant devoir être nuancée. Si la lutte contre les congrégations a certes partiellement pesé sur la définition républicaine de l'association, elle en dit néanmoins davantage sur l'anticléricalisme des républicains alors au pouvoir que sur leur conception définitive de l'association. La loi de 1901, comme l'a justement rappelé Jean-Pierre Machelon, fut en effet « avant tout une loi de combat, présentée et discutée comme telle $»^{21}$. Quelques années plus tard, lorsqu'il revient sur l'action politique du parti radical du début du $\mathrm{XX}^{\mathrm{e}}$ siècle, Ferdinand Buisson la présente d'ailleurs exclusivement comme une «arme» contre le cléricalisme, que la loi 
de séparation de l'Eglise et de l'Etat est entretemps venue compléter avec bonheur ${ }^{22}$.

Sur le moment, les artisans de la loi de 1901 ont en outre reconnu son imperfection en matière associative. Le débat qui se tient par lettres et articles interposés dans le numéro de mai 1901 de la Revue politique et parlementaire est à cet égard significatif. Adversaire - modéré - de la loi, Eugène Rostand signe une étude qui condamne la timidité des républicains. Ils auraient selon lui échoué à donner forme juridique au rôle ambitieux qu'ils avaient jusqu'ici accordé aux «associations ordinaires laïques »: «il ne suffit pas de reconnaittre théoriquement aux citoyens le droit de s'associer, il faut que leurs associations aient le moyen de vivre, de se développer, surtout de durer", écrit-il, accusant à la fois les restrictions apportées à la capacité juridique des associations et le rôle de l'Etat dans l'octroi gradué de la personnalité civile, qui transforme selon lui le droit d'association en «privilège $»^{23}$. Dans les réponses qu'ils publient respectivement en note et à la suite de l'article de Rostand, le fondateur de la revue, Marcel Fournier, et le rapporteur de la loi, Georges Trouillot, tous deux proches de Waldeck-Rousseau, ne nient pas. Ils se contentent de souligner les avancées de la loi par rapport aux textes restrictifs du passé et affirment leur confiance dans l'avenir, qui ouvrira sans doute la voie à de nouveaux approfondissements de la liberté d'association. L'essentiel était ainsi selon Fournier de poser le principe, «qui produira peu à peu ses effets $»^{24}$. «L'avenir dira s'il faut aller plus loin », ajoute Trouillot, plus prudent ${ }^{25}$.

Une question demeure cependant. Si l'obstacle majeur à l'élaboration d'une loi sur les associations résidait jusqu'en 1901 dans la crainte d'accorder aux congrégations les mêmes droits qu'aux associations, pourquoi les républicains ont-ils renoncé à donner aux associations un cadre légal à la mesure de leurs ambitions alors même qu'ils avaient réussi à faire adopter un régime spécial pour les congrégations, développé sous le titre III de la loi ? En d'autres termes, pourquoi avoir défendu une loi qu'ils décrivent eux-mêmes comme inachevée du point de vue des associations alors même que les motifs jusqu'ici invoqués pour justifier leur timidité ont disparu?

\section{UN AUTRE COMPROMIS}

On voudrait ici formuler l'hypothèse selon laquelle l'exclusion des congrégations n'atteint pas l'objectif que les républicains lui avaient 
assigné et que Jean-Paul Martin a justement relevé : tracer une frontière nette entre les «bonnes » et les «mauvaises » associations ${ }^{26}$. Il existe en effet d'innombrables associations religieuses qui ne sont pas des congrégations et qui représentent pourtant à leurs yeux un danger pour la République. Or les républicains sont bien en peine de proposer des critères juridiques qui permettraient de définir a priori la «bonne» association sans remettre en cause la liberté d'opinion: depuis les débuts de la Troisième République, ils ont au contraire pensé leurs propres associations en concurrence avec les associations catholiques, selon les mêmes modes d'organisation et en réponse aux mêmes enjeux, mais pour défendre de tout autres convictions.

Dès 1876, Jean Macé appelle ainsi à lutter contre l'emprise du catholicisme en France en lui empruntant ses propres armes : «Ces messieurs des Cercles catholiques seront sans pitié pour nous s'ils deviennent jamais les maitres. Le plus sage et le plus sûr est de nous mettre, de nos personnes, en travers de leur croisade, et de couvrir, à notre tour, la France entière de Sociétés républicaines ayant pour fin principale de s'opposer à la restauration du Moyen Âge chez nous ${ }^{27} »$. Ces sociétés républicaines devront répondre aux mêmes besoins que les comités catholiques: encadrer l'enfant à la sortie de l'école primaire, pour lui donner « les mêmes avantages que possède celui qui sort des mains de nos adversaires", précise Léon Bourgeois ${ }^{28}$, se «servir» du sentiment d'isolement des étudiants pour leur offrir un refuge propre à consolider en eux l'amour de la République, affirme Paul Bert - «c'est ainsi qu'on a fondé les premiers cercles catholiques d'étudiants ${ }^{29} »$. La mission même des associations républicaines peut être formulée en des termes semblables à ceux que les associations catholiques emploient pour décrire leur propre entreprise. S'adressant aux membres de l'association philotechnique en novembre 1890, Jules Ferry n’hésite pas à paraphraser l'évangile selon Saint-Matthieu : «il vous a été dit, à vous aussi, allez et enseignez, mais ce n'est pas une voix mystique qui vous conduit, c'est le sentiment profond de solidarité humaine et l'amour de l'humanité 30 ».

Qu'est-ce qui distingue alors les comités catholiques des sociétés républicaines, sinon les opinions qu'ils défendent? Et au nom de quoi les républicains pourraient-ils leur refuser la liberté de se développer et de répandre leurs idées? Les républicains n'ont pas éludé ces questions. Dans le célèbre discours qu'il prononce lors de son initiation à la franc-maçonnerie, en 1875, Emile Littré s'interroge ainsi 
sur le combat que se livrent catholiques et républicains pour imposer leur emprise sur l'éducation et l'enseignement: «Est-ce à dire que nous, à notre tour, prétendions en devenir les maitres et la remanier au profit de doctrines que l'on confond souvent avec les doctrines révolutionnaires ? 31 ». Littré bien sûr refuse de réduire l'œuvre républicaine à une lutte de pouvoir analogue à celle des catholiques. Il invoque certes, mais rapidement, la tolérance qui distingue les moyens mis en œuvre par les républicains des pratiques catholiques. Mais ce qui constitue à ses yeux «l'immense différence » entre les entreprises républicaine et catholique, c'est surtout la nature des idées défendues de part et d'autres : «Nous, je l'ai déjà dit, nous acceptons la science telle qu'elle a été et telle qu'elle sera », contrairement aux catholiques, qui, attachés à leurs dogmes, sont voués à l'erreur ${ }^{32}$.

En principe, les associations républicaines ne peuvent donc échouer dans leur entreprise. En diffusant largement la science autour d'elles, elles travaillent à la chute du pouvoir religieux et au triomphe de leurs idées - scientifiques et politiques. Le pays longtemps divisé retrouvera ainsi son unité dans la science et dans la République. Léon Bourgeois le dit nettement : «il y a deux choses qui sont « unes»- la Science et la Nation. La Vérité étant une, il faut que tous ceux qui la professent et la reçoivent sentent l'unité de la science, aient à tous les degrés la même confiance en elle, le même respect pour elle, en reconnaissent partout également la dignité. L'unité des enseignements, conséquence de l'unité de la science, mène à l'unité de la nation. $[\ldots]^{33} \%$.

En pratique cependant, les républicains se divisent. La puissance de la science suffit-elle au triomphe de la République ? Dans ce cas, il faut accorder la liberté pleine et entière à toutes les associations, quelles qu'elles soient: la vérité - républicaine - s'imposera d'ellemême. C'est la position que Jules Simon développe dans son rapport sur la proposition Dufaure: "Oui libres penseurs, il se formera des associations religieuses. Vous n'avez pas le droit de vous y opposer, si vous fondez l'Etat, la République, sur le droit, sur la liberté. Vous n'avez pas le droit de les craindre, si vous croyez à la puissance de la raison et à l'ascendant de la vérité. Avoir peur, c'est n'avoir pas foi ${ }^{34}$ ».

Nombreux sont pourtant les républicains qui craignent que le cléricalisme ne l'emporte, non par la qualité de ses arguments mais en raison du rapport de forces sur le terrain, qui leur parait alors 
défavorable. Comme le souligne Marcel Gauchet, grande était en effet « la crainte, du côté républicain, que 'l'Eglise libre dans l'Etat libre' ne débouche sur 'l'Eglise armée dans l'Etat désarmé' 35 ». Louis Blanc affirme ainsi en 1879 ne pas craindre l'usage que les ennemis de la République pourraient faire de la liberté si les républicains en bénéficient dans la même mesure. Mais ils sont selon lui loin du compte: "Que le clergé cesse de jouir des privilèges qui font sa puissance ; qu'il cesse d'être exempté du service militaire ; qu'il cesse d'être rétribué au moyen d'un impôt auquel contribuent des hommes qui, ne partageant pas ses croyances, n'ont pas recours à ses services : en un mot, qu'il n'y ait plus de société religieuse superposée en quelque sorte à la société civile, et alors, tous étant égaux, liberté pour tous $^{36} »$. Au moment où la loi de 1901 est en discussion à la Chambre et au Sénat, Jules Cabouat ne pose même plus de conditions. La seule présence des adversaires suffit à légitimer la restriction de la liberté pour tous, au nom du danger que représentent quelques-uns : «il serait intolérable que la liberté pût être tournée contre elle-même, servir d'arme de guerre contre l'Etat moderne qui, de plus en plus, représente l'esprit de la Révolution, mettre en péril ses conquêtes et arrêter les réformes nécessaires pour adapter enfin notre organisation politique et sociale aux conditions de vie d'une démocratie sûre de son avenir ${ }^{37} »$.

Au début du XXe siècle, la prudence l'a emporté. La loi de 1901 tranche en faveur d'un développement des associations contrôlé par l'Etat. C'était renoncer à donner aux associations la puissance et l'indépendance qui leur auraient permis de remplir le rôle de garant de l'intérêt général que les républicains leur avaient initialement assigné. Mais la loi avait l'avantage de favoriser les sociétés républicaines, qui obtiendraient plus aisément la reconnaissance d'utilité publique, au détriment des associations religieuses, que la République craignait tant. Si la loi de 1901 représente un compromis, c'est donc moins entre l'individualisme républicain et la reconnaissance du rôle des groupements, ou entre un droit longtemps marqué par la méfiance à l'égard des corps intermédiaires et des pratiques sociales désormais caractérisées par l'association, mais entre des conceptions résolument ralliées à la liberté d'association et la crainte que cette dernière ne serve à renverser la République.

Le détour par les discussions tenues en dehors de l'enceinte parlementaire, au sein des associations ou des revues animées par les 
républicains, aura ainsi permis de donner un nouvel éclairage aux conceptions républicaines de l'association, trop souvent interrogées exclusivement à partir des débats parlementaires qui ont débouché en 1901 sur l'adoption de la loi relative au contrat d'association. Plus ambitieuses en matière associative qu'on ne l'a dit, elles visaient néanmoins un objectif difficile à atteindre, a fortiori en ces temps de lutte anticléricale. Il s'agissait, pour citer une dernière fois le président du Cercle caennais de la Ligue de l'Enseignement, de permettre à la démocratie «de se donner librement à elle-même [une] armature indépendante et vivante mais animée d'un loyalisme à toute épreuve envers la société moderne». Alors seulement, ajoutait-il, " la perpétuité de l'idéal démocratique sera vraiment assurée et mieux défendue que par aucun autre pouvoir contre toute menace, de quelque doctrine ou de quelque parti qu'elle puisse émaner ${ }^{38} »$. Armature "indépendante et vivante mais loyale», telle est la contradiction que le tournant du $\mathrm{XX}^{\mathrm{e}}$ siècle donne à voir au cœur de la conception républicaine de la société civile et dont la loi de 1901 n'aura pas permis de sortir.

\section{NOTES}

[1] Voir notamment Jacqueline Lalouette et Jean-Pierre Machelon (dirs.), Les congrégations hors la loi ? : autour de la loi du 1er juillet 1901, Actes du colloque de Malakoff-Villetaneuse, 27-28 septembre 2001, Paris, Letouzey et Ané, 2002, 304 p.

[2] Jean-Pierre Machelon, «La liberté d'association sous la Troisième République : le temps du refus (1971-1901) », in Claire Andrieu, Gilles Le Béguec et Danielle Tartakowsky (dir.), Associations et champ politique : la loi de 1901 à l'épreuve du siècle, Actes du colloque de Paris, 16 et 17 novembre 2000, Paris, Publications de la Sorbonne, 2001, p. 149.

[3] Pierre Rosanvallon, Le modèle politique français: la société civile contre le jacobisme de 1789 à nos jours, Paris, Éd. du Seuil, 2004, p.337, coll. PointsHistoire.

[4] Claire Andrieu, Gilles Le Béguec et Danielle Tartakowsky (dir.), op. cit. Voir notamment la contribution de Gilles Le Béguec, «Le moment $1901 »$, p. 67-74.

[5] C'est notamment le terme employé par Martine Barthélemy, Associations : un nowvel âge de la participation?, Paris, Presses de Sciences Po, 2000, p. 48 et suiv.

[6] Ibidem, p. 55. 
[7] Jean-Claude Bardout, L'bistoire étonnante de la loi 1901 : le droit des associations avant et après Pierre Waldeck-Rousseau, Lyon, Juris, 2000, 285 p.

[8] Pierre Rosanvallon, op. cit., p. 321 et suiv.

[9] J'ai décrit cet infléchissement de la conception républicaine de la citoyenneté dans ma thèse : La République en quête de citoyens: les républicains francais face au bonapartisme rural (1848-1880), Paris, Presses de Sciences Po, 2010, $368 \mathrm{p}$.

[10] Jules Cabouat, Le droit d'association et la République, Caen, E. Lanier, 1901, p. 22.

[11] Léon Bourgeois, «Le patronage démocratique de la jeunesse et l'esprit de solidarité, discours prononcé à la séance de clôture du XVIe congrès de la Ligue de l'enseignement à Rouen (9 août 1896) », L'Education de la démocratie française: discours prononcés de 1890 à 1896, Paris, E. Cornély, 1897, p. 231.

[12] Yves Guyot, "Le droit d'association: l'association corporative et l'association contractuelle », Revue politique et parlementaire, $n^{\circ} 54$, décembre 1898 , p. 573.

[13] Pour Jean-Paul Martin, la Ligue de l'Enseignement peut être considérée comme le "prototype » des associations républicaines laïques : Jean-Paul Martin, "A la recherche d'un 'modèle associatif laïque' », La revue de l'économie sociale - Espaces et temps associatifs, avril 1988, p.133-143. Voir aussi sa thèse : La Ligue de l'enseignement et la République des origines à 1914, IEP de Paris, 1992, 2 vol.

[14] Léon Bourgeois, «L'œuvre de l'éducation civique et sociale, discours prononcé à Bordeaux au XVe Congrès de la Ligue de l'Enseignement (1895)», op. cit., p. 183.

[15] Pierre Rosanvallon, op. cit., p. 384.

[16] Gilles Le Béguec, art. cit., p. 70.

[17] Charles Floquet, «Discours sur les syndicats professionnels prononcés les 17, 21 et 24 mai 1881 et 19 juin 1883 », Discours et opinions de M. Charles Floquet, publiés par M. Albert Faivre, Paris, Derveaux, 1885, tome 2, p. 122.

[18] «Rapport fait au nom de la commission chargée d'examiner la proposition de loi de M. Dufaure, sur le droit d'association, par M. Jules Simon, sénateur", Journal officiel de la République française - documents parlementaires (Sénat), août 1882, p. 425.

[19] Pierre Waldeck-Rousseau, Associations et congrégations, Paris, E. Fasquelle, 1901, V-452 p.

[20] Pierre Rosanvallon, op. cit., p. 326.

[21] Jean-Pierre Machelon, "Le titre III de la loi du 1er juillet 1901 relative au contrat d'association ", in Jacqueline Lalouette et Jean-Pierre Machelon (dir.), op. cit., p. 50.

[22] Ferdinand Buisson, La politique radicale : étude sur les doctrines du parti radical et radical-socialiste, Paris, V. Giard et E. Brière, 1908, p. 306.

[23] Eugène Rostand, «La loi sur les associations au point de vue des associations ordinaires laïques ", Revue politique et parlementaire, $\mathrm{n}^{\circ} 83$, mai 1901, p. 266

[24] Ibid., p. 263 (en note). 
[25] Georges Trouillot, «La loi sur les associations : réponse à M. Rostand », ibid., p. 278.

[26] Jean-Paul Martin, «La ligue de l'enseignement, la loi de 1901 et le champ politique républicain », in Claire Andrieu, Gilles Le Béguec et Danielle Tartakowsky (dir.), op. cit., p. 468.

[27] Jean Macé, Les idées de Jean-Francois. A cercles catholiques, sociétés républicaines, tome 8, Laon, H. Le Vasseur, 1876, p. 22.

[28] Léon Bourgeois, « Le lendemain de l'école, discours prononcé à Rouen, à l'ouverture du XVIe Congrès de la Ligue de l'Enseignement (6 août 1896)», op. cit., p. 199.

[29] «Lettre adressée par M. Paul Bert au président du cercle des étudiants de Nancy ", in Emile Goutière-Vernolle, Le cercle des étudiants de Nancy, Versailles, Cerf et fils, 1881, p. 6.

[30] Jules Ferry, « Discours du 23 novembre $1890 »$, Discours et opinions de Jules Ferry, Paris, Armand Colin, 1893, tome 7, p. 377.

[31] Emile Littré, Du devoir de l'homme envers lui-même et envers ses semblables, Paris, Chaix, 1906, p.18.

[32] Ibid., p. 20.

[33] Léon Bourgeois, op. cit., p. 207.

[34] «Rapport fait au nom de la commission chargée d'examiner la proposition de loi de M. Dufaure, sur le droit d'association, par M. Jules Simon, sénateur», op. cit., p. 428.

[35] Marcel Gauchet, La religion dans la démocratie : parcours de la laïcité, Paris, Gallimard, 1998, p. 40.

[36] Louis Blanc, «A ceux qui ont foi dans la République. Banquet de Troyes du 18 mai 1879 », Discours politiques 1847-1881, Paris, Germer-Baillière et Cie, 1882, p. 338.

[37] Jules Cabouat, op. cit., p. 3.

[38] Ibid., p. 22. 
Copyright of Tocqueville Review -- La Revue Tocqueville is the property of University of Toronto Press and its content may not be copied or emailed to multiple sites or posted to a listserv without the copyright holder's express written permission. However, users may print, download, or email articles for individual use. 